\title{
6. PHYSICAL OBSERVATIONS OF THE SHORT-PERIOD COMET 1969 IV
}

\author{
K. I. CHURYUMOV and S. I. GERASIMENKO \\ Department of Astronomy, Kiev University, Kiev, U.S.S.R.
}

\begin{abstract}
The new short-period comet Churyumov-Gerasimenko, discovered by the authors on plates taken by the Kiev University cometary expedition to Alma-Ata in September 1969, was systematically photographed with fast telescopes at Byurakan and Alma-Ata until March 1970. Measurements were made of the photographic magnitude of the photometric nucleus, as well as of the photographic and photovisual integral magnitudes. The variations in nuclear magnitude were found to be well correlated with changes in the total sunspot area. The integral photometric parameters are $H_{y}=11.91 \pm 0^{\mathrm{m}} .54$ and $n=4.0 \pm 0.8$ (in the photographic spectral region). Deviations of the tail axis from the prolonged radius vector were considerable. A spectrogram shows the continuum and emission of $\mathrm{CN}, \mathrm{C}_{2}$ and $\mathrm{C}_{3}$ in the head, the continuum and a singlesemission (perhaps $\mathrm{CO}^{+}$) in the tail.
\end{abstract}

During August and September 1969 the authors took part in the third Kiev University expedition to the Alma-Ata Astrophysical Institute (Churyumov and Gerasimenko, 1970). The purpose of the expedition was to carry out visual and photographic searches for new comets in the morning and evening Everhart (1967) zones and also to make photographic observations of the well-known short-period comets Faye (1969 VI), Comas Solá (1969 VIII), Honda-Mrkos-Pajdušáková (1969 V) and the two new comets Kohoutek (1969b) and Fujikawa (1969 VIII). The observations were made with a $50-\mathrm{cm} f / 2.4$ Maksutov telescope and a $17-\mathrm{cm} f / 1$ Schmidt camera. Altogether we took about 100 plates suitable for integral photometry and the determination of exact positions of the above-mentioned comets.

Still in Alma-Ata, we noted on September 20 a cometary object of magnitude 13 on a plate taken September 11 for P/Comas Solá (Figure 1). Back in Kiev on October 22 we found that the object was $1: 8$ from the position of $\mathrm{P} /$ Comas Solá given in the ephemeris. Then we saw $\mathrm{P} / \mathrm{Comas}$ Solá close to its ephemeris position, suggesting that the object we had noted was a new comet. Examination of other plates for P/Comas Solá - two on September 9 and two on September 21 - immediately revealed the new object, and although it was near the edge, it still had its cometary appearance and showed motion among the stars (Figure 2). Vsekhsvyatskij cabled news of our discovery to the IAU Central Bureau for Astronomical Telegrams. The new comet was given the preliminary designation $1969 \mathrm{~h}$, and later the final designation 1969 IV.

On the basis of the first exact positions, reduced by Shmakova (Leningrad) from our measurements, Marsden (U.S.A.) calculated six ephemerides from two parabolic and four elliptical orbits. On October 31 the new comet was photographed by Scovil (U.S.A.). This observation, and then observations by Seki (Japan), Roemer (U.S.A.), and Milet (France), closely confirmed one of the elliptical orbits. Later elements of the elliptical orbit of comet 1969 IV are as follows (Marsden, 1969): 


$$
\begin{aligned}
& T=1969 \text { Sept. 11.029 ET } \\
& \omega=11.192\} \quad a=3.50094 \mathrm{AU} \\
& \Omega=50.353\} \quad 1950.0 \quad e=0.63301 \\
& i=7.145 \quad n^{\circ}=0.150462 \\
& q=1.28483 \mathrm{AU} \quad P=6.55 \mathrm{yr}
\end{aligned}
$$

Thus the new comet proved to be the 73rd member of the Jupiter family of shortperiod comets. The fact that the comet had a close encounter with Jupiter in 1959 (Marsden, 1969) is very interesting. Vsekhsvyatskij (1969) points out that powerful cataclysms take place in the Jupiter system and that this probably led to the production of the new comet one and a half revolutions before its discovery.

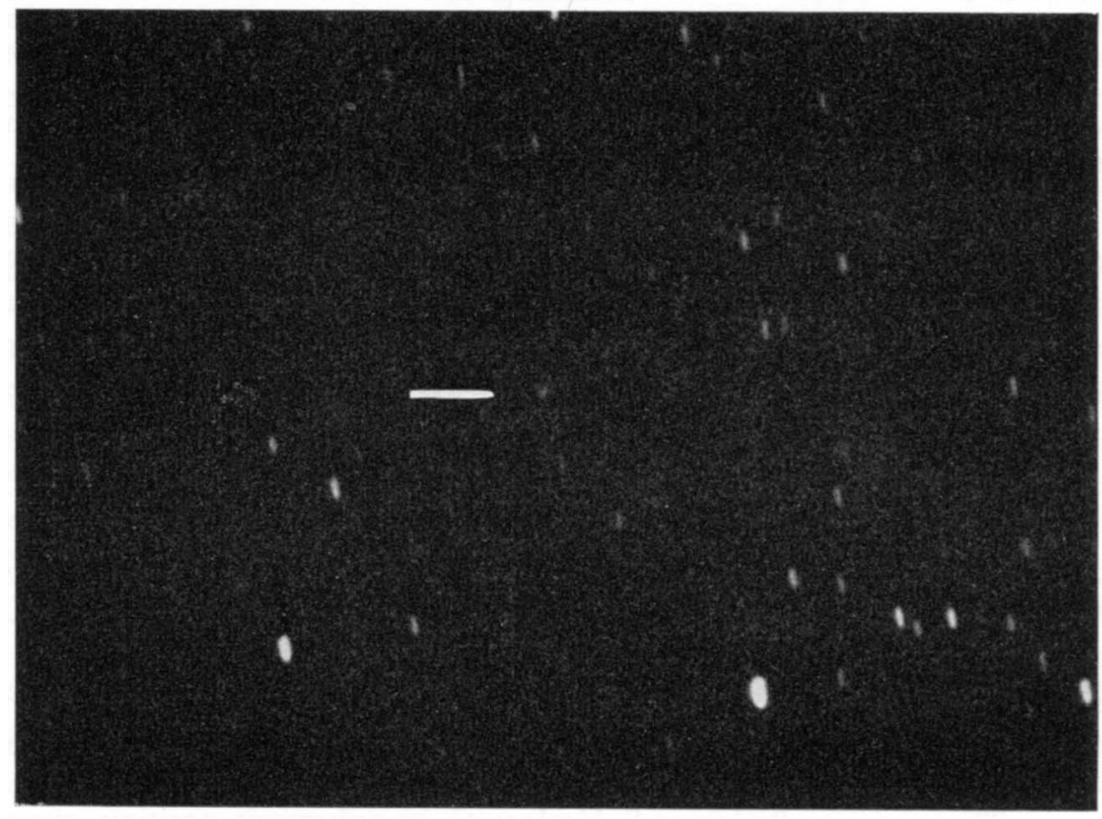

Fig. 1. The plate on which comet 1969 IV was found (1969 Sept. 11.92010 UT).

From the beginning of November 1969 scientists from the Astronomy Department of Kiev University (Vsekhsvyatskij, Gerasimenko, Afanas'ev) systematically observed comet 1969 IV at southern Soviet observatories (Alma-Ata, Byurakan). The purpose of these observations was to obtain a continuous photometric series (which is very important in the case of periodic comets) for determining integral magnitudes of the comet, to study the comet's physical structure and to obtain accurate positions.

During this period visibility conditions made it possible to photograph the comet at low zenith distances (less than $25^{\circ}$ ), which is essential for the photography of faint, extended objects. Most of the material suitable for photometric work was obtained with the $20-\mathrm{cm}$ and $50-\mathrm{cm}$ Schmidt cameras at the Byurakan Astrophysical Observatory (Vsekhsvyatskij, Afanas'ev) and the 50-cm Maksutov telescope at the Alma-Ata 
Astrophysical Institute (Afanas'ev, Gerasimenko, Churyumov). During September 1969-March 1970 more than 50 plates were taken on 34 nights. About 45 plates were

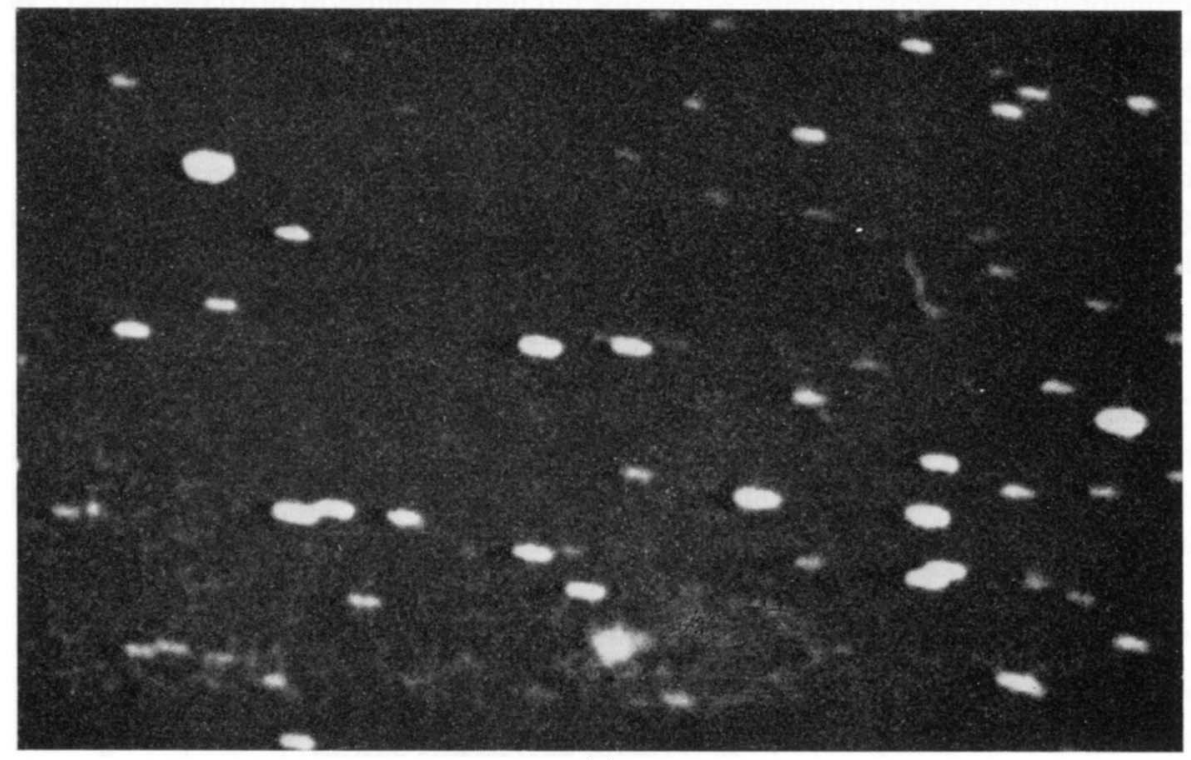

(a)

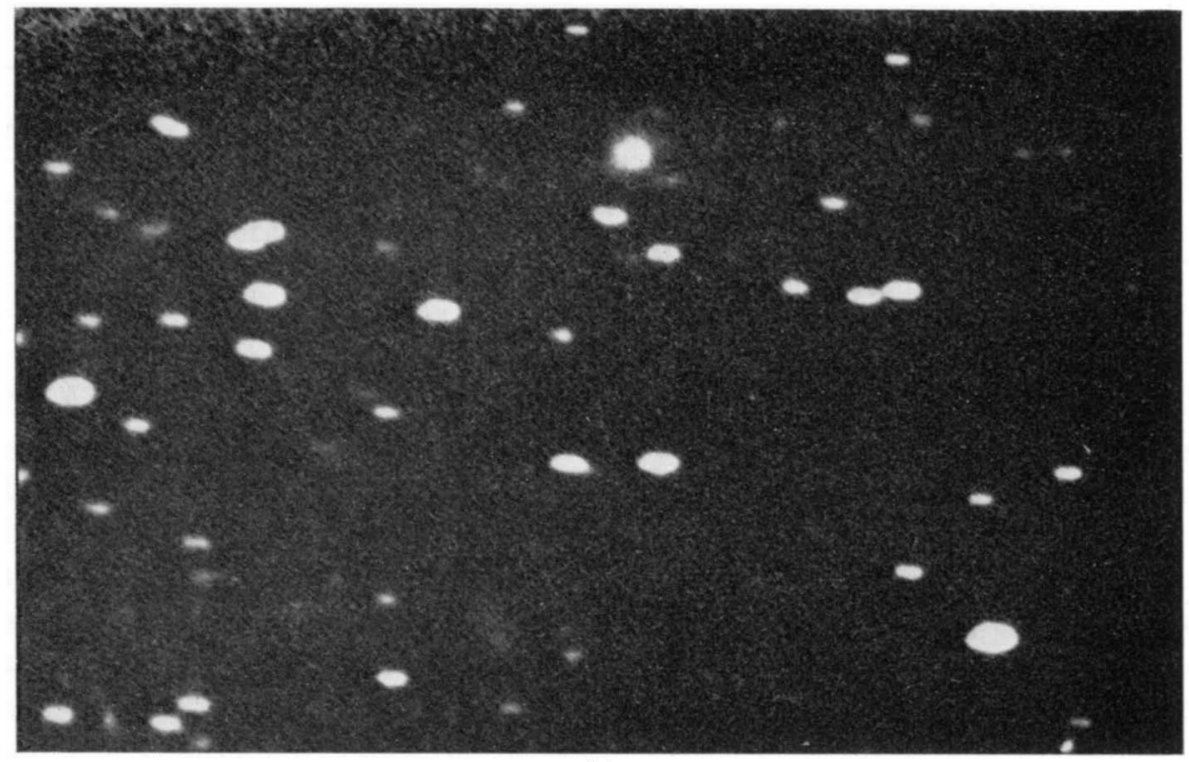

(b)

Fig. 2. Comet 1969 IV (a) 1969 Sept. 21.92752; (b) 1969 Sept. 21.94795.

selected for determining nuclear magnitudes of the comet. Integral photographic magnitudes were obtained from nine plates taken at times of good atmospheric transparency and giving reliable extrafocal standards. 
On most of the plates the comet had a strongly pronounced nucleus $0.06 \mathrm{~mm}$ (50-cm Schmidt) and $0.10 \mathrm{~mm}$ (50-cm Maksutov telescope) in diameter. For our microphotometric measurements we used a circular diaphragm of diameter $0.12 \mathrm{~mm}$ (corresponding to 1 to $2 \times 10^{4} \mathrm{~km}$ at the comet). For standards we obtained extrafocal exposures of star clusters (Coma Berenices, the Pleiades, NGC 2632, NGC 1647, NGC 1628, NGC 2264) and the North Polar Sequence.

From Kodak O-aO and ORWO ZU-2 plates (without filters) we reduced the $B$ magnitudes of the standard clusters to the international photographic system in the following way (Martynov, 1966):

$$
m_{\mathrm{pg}}=-0.18+1.09(B-V)+V .
$$

In Table I, we give $m_{\mathrm{pg}}$ for the photometric nucleus of comet 1969 IV over the entire period of the observations and also the magnitudes $h_{10}$ reduced to unit distances from the Earth and Sun. (On some nights when several plates were taken the average values of $m_{\mathrm{pg}}$ are listed.)

TABLE I

Apparent photographic magnitude $\left(m_{\mathrm{pg}}\right)$ and absolute magnitude $\left(h_{10}\right)$ for the photometric nucleus

\begin{tabular}{|c|c|c|c|c|c|}
\hline 1969 & $m_{\mathrm{pg}}$ & $h_{10}$ & $1969 / 70$ & $m_{\mathrm{pg}}$ & $h_{10}$ \\
\hline \multirow{4}{*}{ Sept. 1} & 14.67 & 12.83 & Dec. 11 & 14.98 & 12.27 \\
\hline & 15.05 & 13.23 & 14 & 13.95 & 11.21 \\
\hline & 14.13 & 12.31 & 15 & 16.02 & 13.25 \\
\hline & & & 16 & 16.09 & 13.31 \\
\hline \multirow{8}{*}{ Nov. } & 14.38 & 11.98 & 19 & 14.55 & 11.74 \\
\hline & 15.32 & 12.92 & 20 & 14.34 & 12.52 \\
\hline & 15.85 & 13.45 & & & \\
\hline & 14.54 & 12.11 & Feb. & 15.60 & 12.08 \\
\hline & 14.45 & 12.01 & 7 & 15.27 & 11.72 \\
\hline & 14.23 & 11.76 & 8 & 15.96 & 12.37 \\
\hline & 16.33 & 13.84 & 9 & 15.72 & 12.06 \\
\hline & & & 13 & 16.23 & 12.46 \\
\hline \multirow[t]{5}{*}{ Dec. } & 14.97 & 12.33 & & & \\
\hline & 15.79 & 13.14 & Mar. 2 & 16.17 & 12.08 \\
\hline & 15.75 & 13.10 & 3 & 16.64 & 12.51 \\
\hline & 15.43 & 12.77 & 4 & 16.44 & 12.29 \\
\hline & 15.22 & 12.53 & 6 & 16.30 & 12.09 \\
\hline
\end{tabular}

In Figure 3 we plot the absolute magnitude $h_{10}$ of the photometric nucleus of the comet and also the sunspot area $S$ against the time. The solar data are taken from Solnechnye Dannye and Solar Phenomena. We took into consideration the difference in heliographic longitude between the Earth and the comet. Since $i=7^{\circ}$, the difference in heliographic latitude is negligible. 
As can be seen from Figure 3, the variations in nuclear magnitude correlate well with the sunspot area changes, except in February. The amplitude of the variations in the brightness of the photometric nucleus was about 2 magnitudes, indicating great activity in the comet. The strongest outburst took place during December 8-14, and the increase in sunspot area at the time was not substantial - although it is possible that there was a considerable output of energetic particles. Besides, it could also be that internal causes of comet activity, such as reactions between radicals produced by the radiation (Donn and Urey, 1957) or explosions of the frozen $\mathrm{NH}$ radical (Rice and Freamo, 1951), play a part.

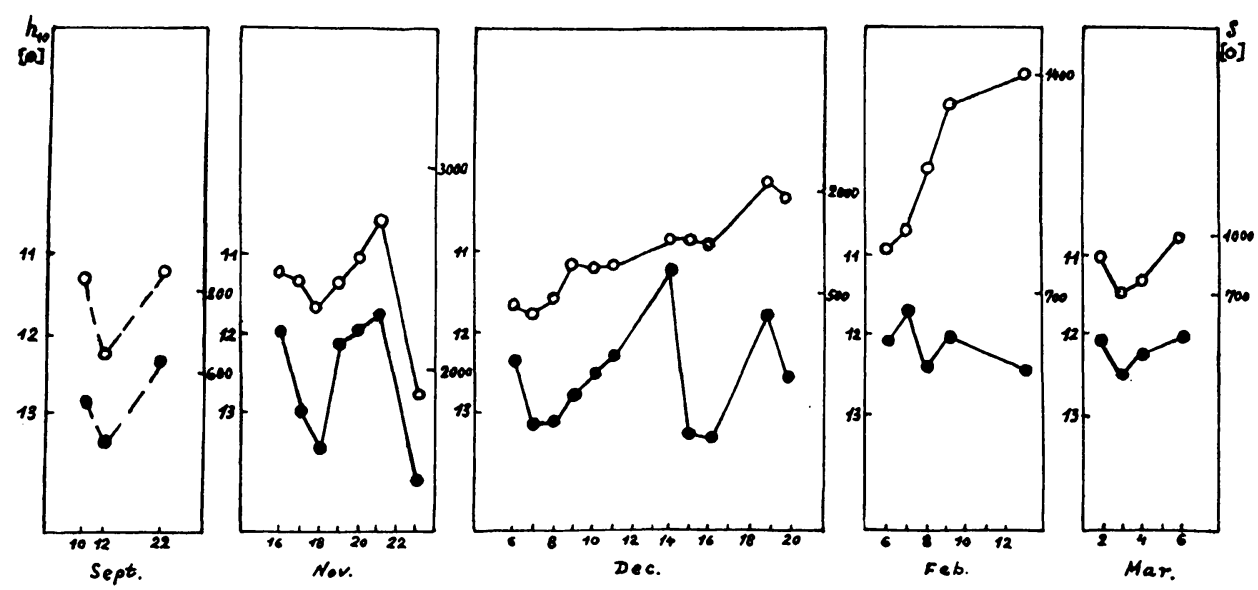

Fig. 3. Variations of the absolute photometric nuclear magnitude $\left(h_{10}\right)$ and the sunspot area $(S)$ with time.

The sensitive response of the comet to variations in the dynamical situation in interplanetary space resulted in the 'synchronous' reaction of the comet's brightness to fluctuations in the solar activity index (especially in November). In accordance with the idea of a quasi-stationary system of corpuscular streams generated in the solar corona (Vsekhsvyatskij et al., 1965) it can be assumed that during the outbursts (e.g., Nov. 18-22, Dec. 8-14 and 16-19, Feb. 6-7 and 8-9, Mar. 3-6) the comet travelled through coronal rays (corpuscular streams) and experienced the increased density and energy of the particles. The action of these particles accelerated the sublimation of cometary ices and the processes of gas dissociation and ionization in the comet's atmosphere. The enhancement of different forms of solar activity (sunspot areas, flares, flocculi) leads to an increase in the total number of the fast particles in the solar corona, and these replenish the coronal rays.

As Vsekhsvyatskij has pointed out, the curves for the photometric nuclear brightness of comet 1969 IV show a tendency for a 27-day recurrence period in the comet 
outbursts: Sept. 22-Nov. 16 (55 days), Nov. 16-Dec. 14 (29 days), Dec. 14-Feb. 7 (54 days), Feb. 7-Mar. 5 (27 days). We also note a 27 -day recurrence period in the minima: Nov. 18, Dec. 15, Feb. 8.

The absence of a difference in time between the nuclear magnitude variations and the changes in the solar activity index can be explained by assuming that the output of particles causing the nuclear outburst does not have to take place at the moment when the active region on the Sun is being generated. To cause an outburst when the comet is 1.5 AU from the Sun, particles travelling with velocities of about 1000 $\mathrm{km} \mathrm{s}^{-1}$ must leave the deepest layers of the photosphere two days before the formation of the active region.

From nine plates Afanas'ev has determined the absolute integral magnitude and the photometric parameters $H_{y}$ and $n$. The results of the measurements of $m_{\mathrm{pg}}$ and $S_{a}$ are given in Table II. $S_{a}$ is the area of the cometary image in square minutes of arc. $H_{y}$ and $n$ were found by least squares to be:

$$
H_{y}=11.91 \pm 0.54, \quad n=4.0 \pm 0.8
$$

The large residuals are an indication of the high activity in the comet. Table III lists some photovisual $(V)$ magnitudes, obtained from plates taken with the Maksutov telescope at Alma-Ata.

\section{TABLE II}

Apparent photographic integral magnitude $\left(m_{\mathrm{pg}}\right)$ and total area of the cometary image $\left(S_{a}\right)$ in square minutes of arc

\begin{tabular}{lrll}
\hline \multicolumn{1}{l}{$1969 / 70$} & $m_{\mathrm{pg}}$ & $S_{a}$ \\
\hline Dec. & 7.06 & 13.95 & 13.1 \\
& 11.08 & 13.33 & 13.1 \\
& 15.04 & 13.88 & 13.6 \\
& 19.08 & 14.46 & 14.2 \\
Feb. & 5.92 & 15.81 & 10.7 \\
& 6.86 & 15.82 & 10.7 \\
& 7.94 & 16.08 & 10.7 \\
& 8.91 & 15.57 & 10.7 \\
& 12.92 & 16.07 & 10.7 \\
\hline
\end{tabular}

Over the entire period of our observations the comet had a narrow, straight tail, probably of type I. Its length ranged from 1' to 11'. On November 16 Burnasheva (Crimean Astrophysical Observatory) noted that there was a fan-like tail. Some characteristics related to the structure of the comet are listed in Table IV, $d$ being the diameter of the coma, $P$ the position angle of the tail, and $s$ the length of the tail. The tail axis deviated from the prolonged radius vector by up to $\pm 20^{\circ}$, which is evidence 
TABLE III

Photovisual $V$ magnitudes

\begin{tabular}{crl}
\hline 1969 & & $V$ \\
\hline Nov. 16.90 & 12.72 \\
& 16.93 & 12.55 \\
& 16.95 & 12.74 \\
& 17.91 & 12.84 \\
& 17.94 & 12.69 \\
Dec. & 6.98 & 12.84 \\
& 7.04 & 13.30 \\
& 7.99 & 13.35 \\
& 8.11 & 13.23 \\
\end{tabular}

TABLE IV

Coma diameter $(d)$, and position angle $(P)$ and length $(s)$ of the tail

\begin{tabular}{|c|c|c|c|c|}
\hline \multicolumn{2}{|c|}{$1969 / 70$} & \multirow{2}{*}{$\frac{d}{1.52}$} & \multirow{2}{*}{$\frac{P}{298.24}$} & \multirow{2}{*}{$\frac{s}{2 ! 4}$} \\
\hline Nov. & 16.90 & & & \\
\hline & 16.93 & 1.43 & 300.90 & 2.4 \\
\hline & 16.95 & 0.99 & 297.05 & 6.3 \\
\hline & 17.91 & 1.72 & 295.82 & 10.6 \\
\hline & 17.93 & 1.28 & 297.24 & 3.5 \\
\hline & 19.08 & 1.11 & 292.20 & 5.5 \\
\hline \multirow[t]{8}{*}{ Dec. } & 6.09 & 0.55 & 306.54 & 4.5 \\
\hline & 6.98 & 1.15 & 288.70 & 4.1 \\
\hline & 7.99 & 1.46 & 301.01 & 9.4 \\
\hline & 8.11 & 1.02 & 300.37 & 11.2 \\
\hline & 10.05 & 0.60 & 301.02 & 1.7 \\
\hline & 11.08 & 0.42 & 295.50 & 7.4 \\
\hline & 16.08 & 1.48 & 303.89 & 3.4 \\
\hline & 19.08 & 0.65 & 305.37 & 3.8 \\
\hline \multirow[t]{4}{*}{ Feb. } & 6.86 & 1.87 & 294.92 & 6.0 \\
\hline & 7.94 & 2.03 & 297.40 & 6.3 \\
\hline & 8.91 & 2.18 & 300.66 & 7.2 \\
\hline & 12.92 & 1.48 & 279.04 & 5.1 \\
\hline Mar. & 1.83 & 0.24 & 293.62 & 4.2 \\
\hline
\end{tabular}

for strong interaction between the comet tail plasma and electromagnetic interplanetary fields (Hoffmeister, 1943).

On November 16 a spectrogram of the comet was obtained by Lipovetskij with the $100-\mathrm{cm}$ Schmidt camera (1.5 prism) at Byurakan. Since the dispersion was very low $(1800 \AA / \mathrm{mm}$ at $\mathrm{H} \gamma)$, only the continuous spectrum of the head and tail was noted. The emissions of $\mathrm{CN}, \mathrm{C}_{2}$ and apparently $\mathrm{C}_{3}$ are visible on a contact print (using the photographic Sabatier effect). Only a single emission becomes visible in the tail (perhaps $\mathrm{CO}^{+}$). We plan to study the spectrum in more detail.

The substantial variations in the nuclear and integral head magnitudes of comet 
1969 IV, the structural changes in the head, and the presence of the long tail are evidence for high activity and therefore the comet's comparative youth.

\section{Acknowledgment}

The authors wish to thank S. K. Vsekhsvyatskij for helpful comments.

\section{References}

Churyumov, K. I. and Gerasimenko, S. I.: 1970, Priroda, Moskva, No. 4.

Donn, B. and Urey, H. C.: 1957, Mem. Soc. Roy. Sci. Liège 18, 124.

Everhart, E.: 1967, Astron. J. 72, 716.

Hoffmeister, C.: 1943, Z. Astrophys. 22, 265.

Marsden, B. G.: 1969, IAU Circ. Nos. 2181, 2187.

Martynov, D. Ya.: 1966, Kurs Prakticheskoj Astrofiziki, Moscow.

Rice, F. O. and Freamo, M.: 1951, J. Am. Chem. Soc. 73, 5529.

Vsekhsvyatskij, S. K.: 1969, in Problemy Sovremennoj Kosmogonii, Moscow.

Vsekhsvyakskij, S. K., Nikolskÿ, G. M., Ivanchuk, V. I., Nesmyanovich, A. T., Ponomarev, E. A., Rubo, G. A. and Cherednichenko, V. I. 1965, Solnechnaya Korona i Korpuskulyarnoe Izluchenie $v$ Mezhplane tom Prostranstve, Kiev. 